\title{
Indications of Transconjunctival Orbital Septum Fat Release and Preservation for Orbitopalpebral Sulcus in Lower Eyelid Blepharoplasty
}

\author{
Jia-Liang Chen $^{1} \cdot$ Chao-Hua Liu ${ }^{1}$ Cheng-Gang Yi ${ }^{1}$
}

Received: 26 February 2017 / Accepted: 1 March 2017/Published online: 23 March 2017

(C) Springer Science+Business Media New York and International Society of Aesthetic Plastic Surgery 2017

Level of Evidence $V$ This journal requires that authors assign a level of evidence to each article. For a full description of these Evidence-Based Medicine ratings, please refer to the Table of Contents or the online Instructions to Authors www.springer.com/00266.

Sir,

We are so happy that our viewpoints of transconjunctival lower eyelid blepharoplasty (TCB) are agreed on by other surgeons. Surely, transconjunctival lower eyelid blepharoplasty is a safe and reliable technique both for excision and repositioning of orbital fat [1]. However, appropriate patient selection is a key element for favorable results. Patients presenting with bulging fat and inferior orbitopalpebral sulcus without apparent dermatochalasis, and skin wrinkles can be corrected through the transconjunctival instead of transcutaneous approach [1], as well as patients without disorders associated with orbicularis muscle paralysis denervated by muscle flaps. In some patients with lid laxity [2] because of redundant eyelid skin and excessive orbital septal fat, the transcutaneous incision was generally used to preserve and release orbital fat, and lift the orbicularis oculi muscle [3,4] for lateral canthal support to reduce the lid malposition. TCB can be performed through the pre- or post-septal approach for the inferior orbitopalpebral sulcus and adjunctive rejuvenation procedures. However, the inferior-oblique-muscle between the interior and lateral orbital fat requires awareness during the approach since its injury may cause diplopia. Once a

Jia-Liang Chen

chenj155@126.com; lailantime@foxmail.com

1 Department of Plastic Surgery, Xijing Hospital, Fourth Military Medical University, 15 Changle Road, Xi'an 710000, Shanxi, China satisfactory contour was achieved, ophthalmic antibiotic ointment was used, and no suture was required for closure to reduce foreign body reaction and corneal irritation, and make-up should be avoided in the early postoperative period. At the same time, preoperative evaluation is important, including eye dryness, lid malposition, diplopia or strabismus, corneal injury, lid laxity, and orbital fat. In summary, transconjunctival lower eyelid blepharoplasty is a reliable technique [5] for patients with visible bulging fat, a significant inferior orbitopalpebral sulcus, but without significant dermatochalasis or skin wrinkles in the lower eyelid.

\section{Compliance with Ethical Standards}

Conflict of interest None.

\section{References}

1. Chen Jia-Liang, Liu Chao-Hua, Yi Cheng-Gang (2017) Transconjunctival orbital septum fat release and preservation for orbitopalpebral sulcus in lower eyelid blepharoplasty. Aesthet Plast Surg. doi:10.1007/s00266-017-0809-z

2. Innocenti A, Mori F, Melita D, Dreassi E, Innocenti M (2016) Effects of orbicularis oculi flap anchorage to the periosteum of the upper orbital rim on the lower eyelid position after transcutaneous blepharoplasty: statistical analysis of clinical outcomes. J Plast Reconstr Aesthet Surg 70:385-391

3. Hamra ST (1995) Arcus marginalis release and orbital fat preservation in midface rejuvenation. Plast Reconstr Surg 96(2):354-362

4. Hamra ST (1996) The use of orbital fat preservation in facial aesthetic surgery a new concept. Clin Plast Surg 23:17-28

5. Sadove RC (2007) Transconjunctival septal suture repair for lower lid blepharoplasty. Plast Reconstr Surg 120:521-529 